\title{
Programa de fluoración de la leche en Codegua, Chile: evaluación al tercer año
}

\author{
Rodrigo Mariño, ${ }^{1,2}$ Alberto Villa ${ }^{1}$ y Sonia Guerrero ${ }^{1}$
}

\begin{abstract}
RESUMEN En 1994 se inició un estudio comunitario para determinar la efectividad del uso de productos lácteos fluorados, distribuidos mediante el Programa Nacional de Alimentación Complementaria, con el fin de lograr una disminución de la caries dental infantil en zonas rurales de Chile en las que esta tiene una alta prevalencia. Se determinaron los índices de prevalencia de caries dental en dos comunidades rurales de la Sexta Región de Chile: una en la que los niños recibieron productos lácteos fluorados (comunidad de estudio: Codegua) y otra en la que recibieron productos lácteos no fluorados (comunidad testigo: La Punta). Después de recibir productos lácteos fluorados durante 3 años, los indices de prevalencia de caries dental presentaron una significativa mejoría en la comunidad de estudio. Al comparar la prevalencia actual de la caries dental en los preescolares de Codegua con la que presentaban antes de la fluoración de los productos lácteos, se comprueba que disminuyó entre 40 y $60 \%$ en niños de 3 a 6 años. En los grupos de 3 y 4 años de edad, la proporción de niños sin antecedentes de caries dental aumentó en $74 \%$ (de 40,7 a 70,8\%) y 71\% (de 33,3 a 56,9\%), respectivamente. Estos resultados, similares a los obtenidos tras la introducción de programas de fluoración del agua potable, se consiguieron sin ningún otro tipo de intervención adicional en el funcionamiento normal del Programa Nacional de Alimentación Complementaria, como podrían ser campañas de motivación de las madres o de educación para el consumo de productos de este programa.
\end{abstract}

La validez y efectividad del flúor en la prevención de la caries dental han sido ampliamente demostradas y en la actualidad hay en diversos países del mundo (1-4) más de 50 años de experiencia con la utilización de diferentes medios como vehículo del flúor $(4,5)$. Uno de ellos ha sido la leche fluorada. Los primeros estudios clínicos en este campo comenzaron en los años 50 en Suiza (6) y demostraron que el consumo de leche fluorada podía reducir

\footnotetext{
1 Departamento de Epidemiología Nutricional, Instituto de Nutrición y Tecnología de los Alimentos, Universidad de Chile, Santiago, Chile.

2 School of Health, University of New England, Armidale, NSW, Australia. La correspondencia debe dirigirse a Rodrigo Mariño a la siguiente dirección postal: School of Health, University of New England, Armidale, NSW 2351, Australia.
}

en $60 \%$ la caries dental en la dentición permanente. Posteriormente, otros estudios clínicos controlados realizados en los Estados Unidos de América (7), Escocia $(8)$ y Hungría $(9,10)$ han confirmado estos resultados.

Sin embargo, muchas veces los beneficios observados en los estudios clínicos controlados no se repiten en situaciones de mayor complejidad, tales como las que se viven en condiciones reales de aplicación en una comunidad $(11,12)$. Durante las dos últimas décadas, la Borrow Dental Milk Foundation ha apoyado en Bulgaria, Chile, China, Perú, Reino Unido y Rusia proyectos comunitarios en los que se ha utilizado la leche como vehículo del flúor (13). En Bulgaria se comenzó a distribuir leche fluorada a 12000 niños de 3 a 10 años de edad en 1988; a los 5 años se obtuvo una reducción de 79 a $89 \%$ en el número de caries en dientes permanentes y de $40 \%$ en dientes temporales (13). En Rusia, a los 3 años se consiguió una reducción de 55 a $68 \%$ en el número de dientes temporales con antecedentes de caries dental en niños de 6 años (14).

En 1994, el Instituto de Nutrición y Tecnología de los Alimentos (INTA) de la Universidad de Chile inició un proyecto de estudio comunitario para demostrar la efectividad de los productos lácteos distribuidos por el Programa Nacional de Alimentación Complementaria (PNAC) como vehículo del flúor. Al comenzar el proyecto se consideró que tendría éxito si, a los 4 años de su instauración, se lo- 
grara una reducción de $30 \%$ en los índices de caries dental de los niños de la comunidad de estudio. El presente artículo describe los resultados preliminares obtenidos a los 3 años de la introducción de este programa de fluoración de la leche.

\section{MATERIALES Y MÉTODOS}

El proyecto se planeó como un estudio comunitario en paralelo con muestras iniciales y finales separadas (15), un tipo de diseño experimental que se utiliza frecuentemente para evaluar los resultados de programas como los de prevención de la caries dental (16-18).

Para este estudio se seleccionaron dos comunidades de la Sexta Región de Chile: Codegua (comunidad de estudio) y La Punta (comunidad testigo). Codegua, con aproximadamente 10500 habitantes en 1994, está situada 100 km al sur de Santiago; La Punta se encuentra $8 \mathrm{~km}$ al norte de Codegua. Ambas poblaciones poseen una actividad económica y características sociodemográficas similares.

En Chile, cada niño es beneficiario, desde su nacimiento hasta los 6 años de edad, de suplementos nutricionales en el ámbito del PNAC, que ha estado operando en los últimos 40 años de forma continua (19). En el PNAC cada niño sigue su dieta normal, pero además recibe leche en polvo, con o sin cereales, según su edad. Estos productos son reconstituidos con agua en el hogar, donde los niños consumen la leche a voluntad. En estas condiciones, el PNAC resultaba un vehículo ideal para la introducción de fluoruros en comunidades en las que no es posible la fluoración del agua.

Teniendo en cuenta los problemas relacionados con la adición de fluoruro de sodio a la leche $(20,21)$ y los estudios de Villa et al. (22) sobre la biodisponibilidad del flúor añadido a la leche en forma de monofluorofosfato de sodio (MFP), se decidió utilizar el MFP como agente fluorante. Su concentración en los productos del PNAC está calculada para proporcionar diariamente una dosis de flúor de $0,25 \mathrm{mg}$ a los niños de 0 a 23 meses de edad; de
$0,5 \mathrm{mg}$ a los de 2 a 3 años, y de 0,75 mg a los de 3 a 6 años. La concentración de MFP en los productos del PNAC se controló periódicamente desde el inicio del estudio, siguiendo la técnica descrita por Kolesnik et al. (23).

A fines de 1994, para determinar el estado inicial de la caries dental, se realizó un estudio basal (año 0) de 177 niños de 3 a 6 años de edad de la comunidad de estudio (Codegua). Partiendo del principio de que la prevalencia de caries dental sería equivalente en las dos comunidades, en esta fase no se estudió la comunidad testigo (La Punta). En abril de 1995 se comenzaron a distribuir mediante el PNAC productos lácteos fluorados a los niños de 0 a 6 años de edad residentes en Codegua. La distribución estuvo a cargo de la nutricionista del consultorio local, como parte de sus funciones normales de trabajo. En La Punta se continuó con la distribución de leche de manera habitual, sin MFP añadido. La ingesta de flúor a través de estos productos fue controlada periódicamente por mediciones de la excreción urinaria del ion en los preescolares de ambas localidades.

En los exámenes del estado de salud bucodental, realizados de acuerdo con la metodología y recomendaciones de la Organización Mundial de la Salud (24), con los niños recostados y con iluminación natural, se midieron las superficies cariadas, obturadas o perdidas por caries, lo cual permitió calcular los índices cpod y $\operatorname{cpos}^{3}$ en la dentición temporal. Tanto la fiabilidad interobservadores como la fiabilidad intraobservador, determinadas por el índice kappa, fueron superiores a 0,9 , lo cual refleja una concordancia casi perfecta (25).

El tamaño muestral mínimo, 50 individuos de cada comunidad por cada grupo de edad (3, 4, 5 ó 6 años), se determinó según los criterios de Cohen (26) para detectar un tamaño de efecto "mediano" [(media inicial - media

\footnotetext{
El índice cpod es la media aritmética del número de dientes $(d)$ cariados $(c)$, perdidos $(p)$ u obturados (o) a consecuencia de la caries en dientes temporales (de leche). El índice cpos es similar, pero utiliza como unidad de medida la superficie dental (s), en vez del número de dientes. Los índices equivalentes para los dientes permanentes se abrevian CPOD y CPOS
}

final) /desviación estándar $=0,50]$, en una prueba unilateral con un nivel de confianza de $95 \%$ y una potencia de $80 \%$. Los participantes fueron seleccionados por métodos no probabilísticos intencionados. Para ello, se acudió a las escuelas primarias y jardines de infancia de ambas comunidades y se examinó a todos los niños presentes hasta completar el número necesario.

Los resultados se presentan de tres maneras diferentes. Primero, se realizó una comparación retrospectiva, sin grupo testigo; es decir, se compararon los índices cpod y cpos del año 0 con los del año 3 en cada grupo de edad de la comunidad de estudio. De esta manera, las muestras iniciales y finales pueden considerarse muestras independientes (15). En segundo lugar, se compararon los índices obtenidos al tercer año del estudio en una y otra comunidad. Finalmente, se tomó al individuo como unidad de estudio y se calcularon los porcentajes de niños sin antecedentes de caries dental, independientemente del número de dientes comprometidos o de la gravedad del compromiso; dichos porcentajes fueron comparados tanto retrospectivamente (entre los años 0 y 3 ) en la comunidad de estudio como entre esta y la comunidad testigo a los 3 años. Las diferencias entre los índices de caries se analizaron con la prueba de MannWhitney y las diferencias entre las proporciones de niños sin antecedentes de caries con la prueba de $j i$ al cuadrado $\left(\chi^{2}\right)$; el nivel de significación estadística se fijó en 0,05 para ambas pruebas. Un análisis preliminar indicó que no existían diferencias significativas en función del sexo de los participantes, de modo que en los análisis subsiguientes se agruparon los datos de ambos sexos en cada grupo de edad.

El protocolo experimental del estudio fue aprobado por el comité de ética del INTA y los padres de los niños participantes otorgaron por escrito su consentimiento informado.

\section{RESULTADOS}

Siguiendo las especificaciones de tamaño y método muestral, en abril de 
1998 se examinaron 194 niños de 3 a 6 años de edad en Codegua y 201 de las mismas edades en La Punta. Debido a dificultades para encontrar niños de los grupos de más corta edad y a limitaciones de tiempo y recursos, el número de participantes en algunos grupos de edad fue marginalmente inferior al proyectado $(n=50)$.

El cuadro 1 revela que, entre los años 0 y 3, el índice cpod sufrió en la comunidad de estudio reducciones estadísticamente significativas que oscilaron entre 40 y $46 \%$, dependiendo del grupo de edad. Lo mismo ocurrió con el índice cpos, cuyas reducciones fueron de 48 a $61 \%$.

En el cuadro 2, que compara las dos comunidades al tercer año del estudio, se observa una tendencia general a la disminución de los dos índices de caries (cpod y cpos) en la comunidad de estudio. Las diferencias fueron estadísticamente significativas para ambos índices en los grupos de 3 y 4 años de edad; en los de 5 y 6 años no llegaron a alcanzar el nivel de significación estadística, aunque los valores obtenidos siguieron siendo más favorables en la comunidad de estudio, con la única excepción del índice cpos en el grupo de 6 años.

En el cuadro 3, la comparación entre los años 0 y 3 en la comunidad de estudio muestra un aumento significativo de los porcentajes de niños sin antecedentes de caries dental en todos los grupos de edad, excepto en el de 5 años. En la comparación de ambas comunidades al tercer año también se observan en todos los grupos de edad mejores resultados en Codegua, aunque las diferencias solo fueron estadísticamente significativas para los niños de 3 y 4 años.

\section{DISCUSIÓN}

Estos resultados preliminares, obtenidos a los 3 años del comienzo de un estudio comunitario sobre la efectividad de los productos lácteos del PNAC como vehículo del flúor, revelan que, utilizando la leche como vehículo del agente fluorante (MFP), es posible obtener una disminución significativa de

CUADRO 1. Índices cpod y cpos por edades en la comunidad de estudio (Codegua): comparación entre los años 0 y 3 del estudio. Codegua, Chile, 1994-1998

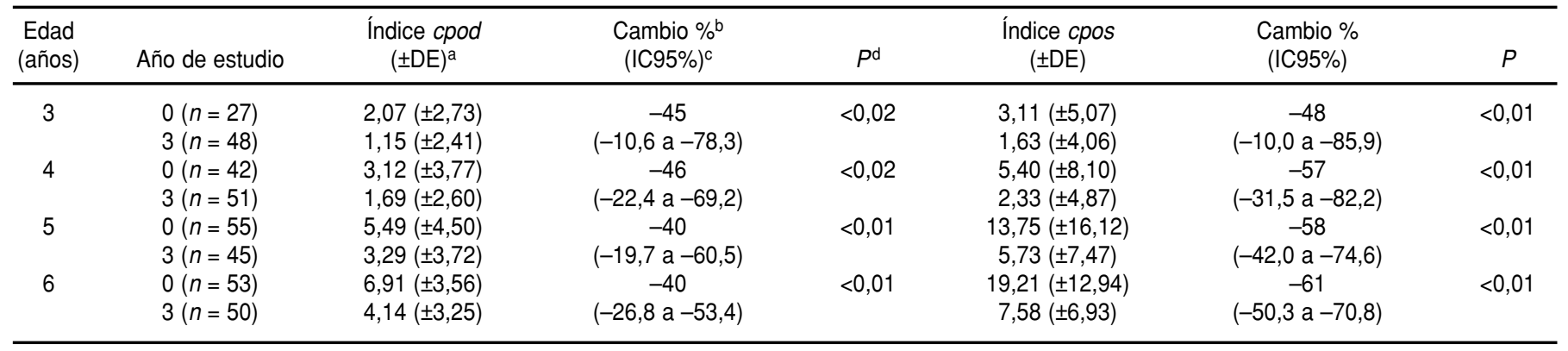

a Desviación estándar.

b Cambio porcentual de los índices.

c Intervalo de confianza de $95 \%$

d Prueba de Mann-Whitney.

CUADRO 2. Índices cpod y cpos por edades en el año 3 del estudio (1998): comparación entre las comunidades de estudio (Codegua) y testigo (La Punta). Codegua y La Punta, Chile, 1998

\begin{tabular}{|c|c|c|c|c|c|c|c|}
\hline $\begin{array}{l}\text { Edad } \\
\text { (años) }\end{array}$ & Comunidad & $\begin{array}{c}\text { Índice cpod } \\
\quad( \pm D E)^{a}\end{array}$ & $\begin{array}{l}\text { Cambio \%b } \\
(I C 95 \%)^{c}\end{array}$ & $P^{d}$ & $\begin{array}{l}\text { Índice cpos } \\
\qquad( \pm D E)\end{array}$ & $\begin{array}{c}\text { Cambio \% } \\
\text { (IC95\%) }\end{array}$ & $P$ \\
\hline \multirow[t]{2}{*}{3} & La Punta $(n=46)$ & $2,30( \pm 3,08)$ & -50 & \multirow[t]{2}{*}{$<0,01$} & $2,89( \pm 4,58)$ & -44 & \multirow[t]{2}{*}{$<0,01$} \\
\hline & Codegua $(n=48)$ & $1,15( \pm 2,41)$ & $(-20,0 \mathrm{a}-80,4)$ & & $1,63( \pm 4,06)$ & $(-3,1 \mathrm{a}-84,4)$ & \\
\hline \multirow[t]{2}{*}{4} & La Punta $(n=41)$ & $3,73( \pm 3,18)$ & -55 & \multirow[t]{2}{*}{$<0,01$} & $5,17( \pm 4,95)$ & -55 & \multirow[t]{2}{*}{$<0,01$} \\
\hline & Codegua $(n=51)$ & $1,69( \pm 2,60)$ & $(-35,1 \mathrm{a}-74,3)$ & & $2,33( \pm 4,87)$ & $(-28,4 a-81,4)$ & \\
\hline \multirow[t]{2}{*}{5} & La Punta $(n=63)$ & $4,00( \pm 3,69)$ & -18 & \multirow[t]{2}{*}{$\mathrm{NS}^{\mathrm{e}}$} & $6,95( \pm 8,18)$ & -18 & \multirow[t]{2}{*}{ NS } \\
\hline & Codegua $(n=45)$ & $3,29( \pm 3,72)$ & $(10,3 \mathrm{a}-45,8)$ & & $6,73( \pm 7,47)$ & $(14,8 \mathrm{a}-49,8)$ & \\
\hline \multirow[t]{2}{*}{6} & La Punta $(n=51)$ & $4,80( \pm 4,51)$ & -14 & \multirow[t]{2}{*}{ NS } & $7,41( \pm 9,03)$ & 2,3 & \multirow[t]{2}{*}{ NS } \\
\hline & Codegua $(n=50)$ & $4,14( \pm 3,25)$ & $(5,4 a-32,9)$ & & $7,58( \pm 6,93)$ & $(28,9 \mathrm{a}-24,3)$ & \\
\hline
\end{tabular}

a Desviación estándar.

b Cambio porcentual de los índices.

c Intervalo de confianza de 95\%.

d Prueba de Mann-Whitney.

e No significativo. 
CUADRO 3. Porcentajes de niños sin antecedentes de caries dental por edades: comparaciones entre los años 0 y 3 en la comunidad de estudio (Codegua) y entre las comunidades de estudio y testigo (La Punta) al tercer año del estudio. Codegua y La Punta, Chile, 1994-1998

\begin{tabular}{|c|c|c|c|c|c|c|}
\hline \multirow[b]{2}{*}{$\begin{array}{l}\text { Edad } \\
\text { (años) }\end{array}$} & \multicolumn{3}{|c|}{ Codegua } & \multicolumn{3}{|c|}{ Año 3} \\
\hline & $\begin{array}{c}\text { Año } 0 \\
(\%)\end{array}$ & $\begin{array}{c}\text { Año } 3 \\
(\%)\end{array}$ & $P^{a}$ & $\begin{array}{l}\text { La Punta } \\
(\%)\end{array}$ & $\begin{array}{c}\text { Codegua } \\
(\%)\end{array}$ & $P$ \\
\hline 3 & 40,7 & 70,8 & $<0,02$ & 45,7 & 70,8 & $<0,02$ \\
\hline 4 & 33,3 & 56,9 & $<0,05$ & 29,3 & 56,9 & $<0,01$ \\
\hline 5 & 21,8 & 37,8 & $N S^{b}$ & 22,2 & 37,8 & NS \\
\hline 6 & 3,8 & 22,0 & $<0,01$ & 19,6 & 22,0 & NS \\
\hline
\end{tabular}

a Prueba de $j i$ al cuadrado $\left(x^{2}\right)$.

${ }^{\mathrm{b}}$ No significativo.

los índices de caries en la dentición temporal y un significativo aumento del porcentaje de niños sin antecedentes de caries dental entre los que habían nacido o tenían alrededor de 1 año de edad al comenzar el programa. Estas diferencias se obtuvieron al comparar la comunidad de estudio tanto consigo misma al inicio del programa como con la comunidad testigo al tercer año. En cambio, en la comunidad testigo, todos los grupos de edad, incluidos los de 3 y 4 años, presentaron índices de caries muy similares a los registrados en el estudio basal.

En cuanto a los porcentajes de niños sin antecedentes de caries dental, en la comunidad de estudio se observó un aumento de $74 \%$ (recorrido de 40,7 a $70,8 \%$ ) en el grupo de 3 años y de $71 \%$ (recorrido de 33,3 a 56,9\%) en el de 4 años. En cambio, en la comunidad testigo las cifras del tercer año seguían siendo similares a las del año 0. La importancia de esto radica en el hecho de que los niños de 3 y 4 años de edad de Codegua, pero no los de La Punta, se encuentran en condiciones de alcanzar la meta de la Organización Mundial de la Salud, que se propone conseguir en el año 2000 que la mitad de los niños de 5 años de edad no tengan antecedentes de caries dental (3).

Estos resultados son similares a los obtenidos en la dentición temporal por otros estudios $(9,10)$ que, a los 5 años de la introducción de un programa de fluoración de la leche, mostraron una disminución de $40 \%$ en los índices de caries en la dentición temporal. Por otra parte, un informe del Ministerio de Salud de Chile (27) publicado 9 años después del inicio de un programa de fluoración del agua potable en Valparaíso reveló que $43 \%$ de los niños de 4 a 5 años de edad no tienen antecedentes de caries, lo cual representa un aumento de $71,2 \%$ con respecto a los valores basales de Valparaíso. Otro estudio (28), con datos específicos para la edad de 3 años en niños que nacieron después del inicio de la fluoración del agua, reveló que, tras 6 años de fluoración del agua potable en Valparaíso, $58,5 \%$ se encuentran libres de caries dental. Comparando estos datos con los obtenidos en Codegua al tercer año del estudio, se comprueba que, a los 3 y 4 años se han logrado proporciones equivalentes de niños sin antecedentes de caries en Codegua y en Valparaíso.

Por último, es de destacar que estos resultados se obtuvieron sin ninguna intervención administrativa o técnica en el funcionamiento del PNAC en Codegua. Estos atributos prácticos y la efectividad de los productos lácteos del PNAC como vehículo para hacer llegar floruros a la comunidad sugieren el uso de este método en la prevención de la caries a nivel comunitario en zonas donde no se puedan utilizar otros vehículos de fluoruros, tales como el agua potable o la sal.

Agradecimiento. El estudio, auspiciado por la Organización Mundial de la Salud, fue financiado por la Borrow Dental Milk Foundation, contó con el apoyo técnico del Departamento Odontológico del Ministerio de Salud de Chile y se realizó mediante un convenio de cooperación con la Dirección de Salud de la VI Región y la Ilustre Municipalidad de Codegua, Chile.

\section{REFERENCIAS}

1. American Dental Association. Accepted dental therapeutics. $39^{\mathrm{a}}$ ed. Chicago: ADA; 1982

2. American Dietitian Association. Position of the American Dietitian Association: the impact of fluoride on dental health. J Am Diet Assoc 1994;94:1428-1431.

3. World Health Organization. Prevention methods and programs for oral diseases. Geneva: WHO; 1984. (Technical report series 713).

4. Burt BA, Fejerskov O. Water fluoridation. En: Fejerskov O, Ekstrand J, Burt BA, eds. Fluoride in dentistry. Copenhagen: Munksgaard; 1996. pp. 275-290.
5. World Health Organization. Fluorides and oral health. Geneva: WHO; 1994. (Technical report series 846).

6. Ziegler E. Bericht über den winterhurer grossversuch mit fluorzugabe zur haushaltmilch. Helv Paediatr Acta 1964;19:343-354

7. Russoff LL, Konikoff BS, Frye JB, Johnston JE, Frye WW. Fluoride addition to milk and its effect on dental caries in school children. Am J Clin Nutr 1962;11:94-101.

8. Stephen KW, Boyle IT, Campbell D, McNee S, Boyle P. Five-year double-blind fluoridated milk study in Scotland. Community Dent Oral Epidemiol 1984; 12:223-229.
9. Bánóczy J, Zimmermann P, Hadas E, Pinter A, Bruszt V. Effect of fluoridated milk on caries: 5 years results. J R Soc Health 1985;105(3): 99-103.

10. Gyurkovics C, Zimmerman P, Hadas E, Bánóczy J. Effect of fluoridated milk on caries: 10 years results. J Clin Dent 1992; 3:121-124.

11. O'Mullane DM. Efficiency in clinical trials of caries preventive agents and methods. Community Dent Oral Epidemiol 1976;4:190-194.

12. Flay BR, Phil D. Efficacy and effectiveness trials (and other phases of research) in the development of health promotion programs. Prev Med 1986;15:451-474. 
13. Borrow Dental Milk Foundation. Annual review. Portsmouth-England: Borrow Dental Milk Foundation; November 1996.

14. Borrow Dental Milk Foundation. Annual review. Portsmouth-England: Borrow Dental Milk Foundation; October 1998.

15. Cook TD, Campbell DT. Quasi-experimentation. Boston: Houghton Mifflin Company; 1979.

16. Lilienfeld AM, Lilienfeld DE. Foundations of epidemiology. $2^{\mathrm{a}}$ ed. New York: Oxford University Press; 1980.

17. Spencer JA. Skewed distributions-new outcome measures. Community Dent Oral Epidemiol 1997;25:52-59.

18. Margetts BM, Nelson H. Design concepts in nutritional epidemiology. Oxford New York: Oxford University Press; 1991.

19. Mardones F, González N, Mardones R, Salinas J, Albala C. Programa Nacional de Alimentación Complementaria en Chile en el período 1937-1982: Rev Chil Nutr 1986;14: $137-182$
20. Ekstrand J, Ehrnebo M. Influence of milk products on fluoride availability in man. Eur J Clin Pharmacol 1979;16:211-215.

21. Duff EJ. Total and ionic fluoride in milk. Caries Res 1981;15:406-408.

22. Villa A, Guerrero S, Cisternas P, Monckeberg F. Fluoride bioavailability from disodium monofluorphosphate fluoridated milk in children and rats. Caries Res 1989;23:179-183.

23. Kolesnik AK, Phillips PC, Villa AE. Physicochemical studies on milk fluoridation. En: Stephen K, Bánóczy J, Pakhomov GN, eds. Milk fluoridation for the prevention of dental caries. Geneva: WHO; 1996. pp. 10-26. (WHO/ $\mathrm{ORH} / \mathrm{MF} / \mathrm{DOC} 96.1$ ).

24. World Health Organization: Oral health survey: basic methods. $3^{\mathrm{a}}$ ed. Geneva: WHO; 1987.

25. World Health Organization: Calibration of examiners for oral health epidemiological survey. The oral health programme. Geneva: WHO; 1993 (Publicación ORH/EIS/EPID.93.1).
26. Cohen, J. Statistical power analysis for the behavioral sciences. Revised ed., New York: Academic Press Inc.; 1977.

27. Chile, Ministerio de Salud: Análisis descriptivo-comparativo de la salud bucal de los grupos etáreos significativos de la $\mathrm{V}$ Región con programa de fluoración del agua potable. Ministerio de Salud 1984-1989. Santiago: Ministerio de Salud; 1994.

28. Mariño R, Onetto JE. Caries experience in urban and rural Chilean 3-year-olds. Community Dent Oral Epidemiol 1995;23:60-61.

Manuscrito recibido el 26 de octubre de 1998 y aceptado para publicación, tras revisión, el 18 de marzo de 1999.
ABSTRACT

\section{The milk fluoridation program in Codegua, Chile: an evaluation after 3 years}

A study was done in 1994 to determine the effectiveness of the fluoridated dairy products that the Chilean National Program for Supplementary Feeding had been distributing to reduce the high prevalence of children's dental caries in rural areas of that country. For the study, the prevalence of caries was assessed in two rural communities of the Sixth Region of Chile. Children in the study community of Codegua had received fluoridated dairy products, while children in the control community of La Punta had received nonfluoridated dairy products. Three years after the program began in Codegua, the community showed a significant improvement in the indices of prevalence of caries. Over that time period, the prevalence of caries among children 3 to 6 years old declined between $40 \%$ and $60 \%$. Among children 3 years old, the proportion of them without a history of caries increased by $74 \%$, from $40.7 \%$ to $70.8 \%$. Among 4 -year-olds, that proportion rose by $71 \%$, from $33.3 \%$ to $56.9 \%$. Similar to results obtained elsewhere with programs to fluoridate drinking water, the outcomes in Codegua were achieved without any other steps by the National Program for Supplementary Feeding, such as motivational campaigns for mothers or educational efforts to encourage consumption of the program's food products. 